\title{
In vivo imaging of pulmonary alveolar proteinosis using confocal endomicroscopy
}

\section{To the Editors:}

Fibred confocal fluorescence microscopy (FCFM) is a new technology that can provide microscopic imaging of a living tissue through a 1-mm flexible fibreoptic miniprobe [1, 2]. To image the alveolar structures in vivo, the miniprobe is advanced through the working channel of the bronchoscope down to the alveolar ducts and sacs. In nonsmoking subjects, the FCFM is able to image the peripheral elastin framework of the alveolar ducts [3]. In active smokers, the technique also images fluorescent alveolar macrophages which contain tobacco tar [3]. The technique has the potential to improve the in vivo diagnosis of peripheral lung diseases during bronchoscopy.

Herein we report the use of FCFM during bronchoscopy on a smoking patient presenting with diffuse alveolar and interstitial opacities on chest radiograph. The in vivo confocal fluorescence endomicroscopy in real-time showed the globular lipoproteinaceous material which is typical of pulmonary alveolar proteinosis (PAP). This diagnosis was confirmed by cytological analysis of the bronchoalveolar lavage (BAL) fluid, and by the presence of high levels of anti-granulocyte-macrophage colony-stimulating factor (GM-CSF) serum antibodies.

This is the first report of the in vivo confocal microscopic appearance of lipoproteinaceous material during bronchoscopy, which appears to be typical of PAP.

A 42-yr-old, 25 pack-yr, active smoking male presented with a 4-month history of increasing breathlessness on exertion and dry cough. He reported a history of heavy and short household plaster dust exposure preceding the onset of symptoms. The physical examination was unremarkable. The chest radiograph showed bilateral alveolar and interstitial opacities. A highresolution computed tomography (CT) scan of the chest showed bilateral ground-glass opacities with interlobular septa thickening, creating a mosaic or "crazy paving" pattern in both lungs (fig. 1). Pulmonary function tests showed a restrictive ventilatory defect, with forced vital capacity at $54 \%$ and diffusion capacity at $37 \%$ of the predicted values. Blood and differential leukocyte counts were in the normal range.

A confocal fluorescence bronchoalveoscopy was performed after nasopharyngeal topical anaesthesia, as described previously $[1,3]$. A 4.4-mm flexible bronchoscope (MP60 model; Olympus Corporation, Tokyo, Japan) was introduced into the airways and distally blocked into a sub-segment of the right middle lobe. In vivo alveolar confocal imaging was performed using the $488 \mathrm{~nm}$ Cellvizio ${ }^{\circledR}$ lung device (Mauna Kea Technologies, Paris, France) and a 1-mm flexible confocal Alveoflex ${ }_{\circledR}$ miniprobe according to the manufacturer recommendations (Mauna Kea Technologies). The miniprobe was introduced into the working channel of the bronchoscope and gently pushed down until the alveolar structure could be recognised. No exogenous fluorophore was used. In five out of the six alveolar areas explored in vivo from the right middle and right lower lobe, the fluorescence confocal imaging showed alveolar filling with 100-300 $\mu \mathrm{m}$ highly fluorescent globular and granular structures, floating into a slightly fluorescent alveolar fluid with preserved acinar elastic structures and fluorescent alveolar macrophages (fig. 2, online supplementary material). After this procedure $150 \mathrm{~mL}$ of saline serum BAL was performed in the right middle lobe. The BAL fluid had a normal macroscopic appearance. No side-effects were encountered during or after the procedure. The chest radiograph performed immediately after the bronchoscopy was unchanged. The BAL cytological examination showed amorphous, granular, eosinophilic, periodic acid-Schiff (PAS)-positive material similar to the structures observed in vivo, leading to the diagnosis of PAP [4]. The epifluorescence microscopic analysis of the BAL fluid confirmed that the fluorescent structures imaged in vivo corresponded to lipoproteinaceous material (fig. 2). The diagnosis of autoimmune PAP was further supported by the presence of a high titre of anti-GM-CSF antibody in the serum (titre: $6 \times 10^{2}$, TF1 cell line growth inhibition method; Immunology Dept, Rennes University Hospital, Rennes, France).

PAP is a rare pathological condition characterised by an accumulation of surfactant which fills the terminal airways and alveoli, thereby impairing gas exchange and engendering respiratory insufficiency [5]. The vast majority of the primary forms of PAP in adults are due to a defect in the clearance of surfactant within alveolar macrophages because of autoantibodies targeting GM-CSF in blood and tissues, including pulmonary alveoli [6].

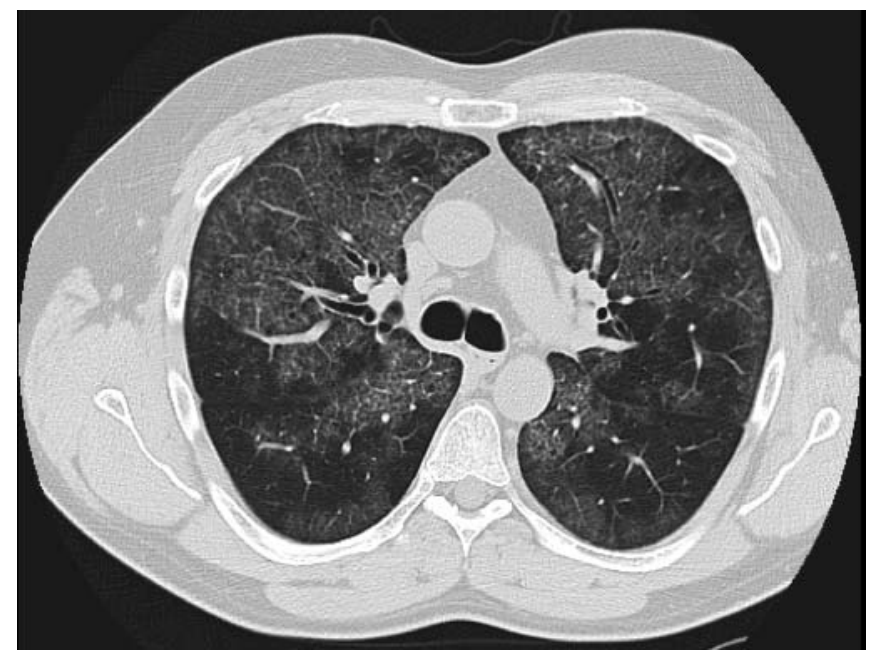

FIGURE 1. A high-resolution computed tomography scan of the chest shows patchy areas of ground-glass opacities and interlobular septa thickening, a pattern commonly characterised as "crazy paving". 

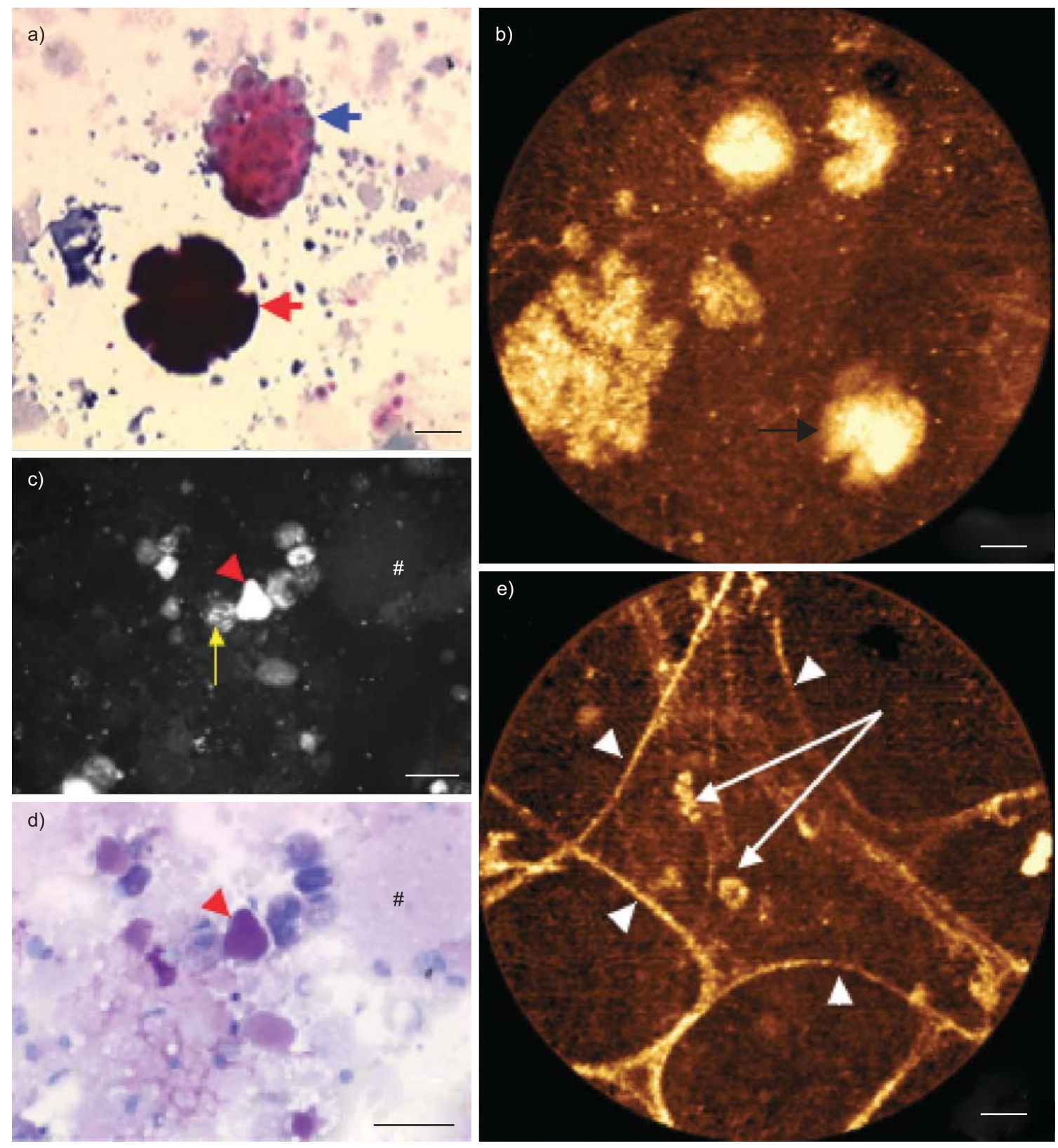

FIGURE 2. a) Eosin and azure B staining of the patient's bronchoalveolar lavage (BAL) fluid shows clustered alveolar macrophages (blue arrow) and characteristic globular lipoproteinaceous material (red arrow). b) In vivo alveolar fibred confocal fluorescence imaging at $488 \mathrm{~nm}$ shows similar highly fluorescent globular structures (black arrow), with well-defined edges and homogeneous fluorescence. c) Epifluorescence imaging at $488 \mathrm{~nm}$ and d) periodic-acid Schiff (PAS) coloration of the same BAL fluid shows highly autofluorescent and PAS-positive globular material (red arrowhead). The flocculent material (\#) appears weakly fluorescent and weakly PAS positive. Fluorescent alveolar macrophages display scattered fluorescent spots (yellow arrow). e) Fibred confocal fluorescence imaging of a normal alveolar area from the patient shows typical alveolar macrophages (white arrow) and normal alveolar elastin fibres of the alveolar duct (arrowheads). The alveolar macrophages can easily be distinguished from the lipoproteinaceous material (b) as they are smaller and display heterogeneous fluorescence. Scale bars $=50 \mu \mathrm{m}$.

The diagnosis of PAP is usually suspected on examination of the thoracic CT scan, which usually displays patchy groundglass opacities with superimposed interlobular septa and intralobular thickening, leading to a crazy paving pattern [7]. However, these signs are not specific and may be observed in a range of other diseases, such as lymphangitic carcinomatosis and acute respiratory distress syndrome [8]. An opalescent, milky, BAL fluid may also be suggestive of the diagnosis, but appears inconstant as in our observation [6]. A definite diagnosis requires the demonstration of typical findings such as the presence of granular acellular eosinophilic lipoproteinaceous material either on the cytological analysis of PASstained BAL fluid, or on open or transbronchial lung biopsy [6]. The presence of a high titre of anti-GM-CSF autoantibodies in the serum usually confirms the autoimmune nature of the disease [9]. 
In this observation, the fluorescence confocal microimaging of the distal lung displayed characteristic PAP globular structures that appeared highly fluorescent in vivo. We reviewed 173 alveolar areas explored with confocal fluorescence endomicroscopy from 24 smoking non-PAP subjects, including nine patients with various interstitial lung diseases (ILD) (sarcoidosis $n=1$; respiratory bronchiolitis-interstitial lung disease $n=4$; asbestosis $n=1$; systemic sclerosis $n=1$; histiocytosis $n=1$; and cryptogenic organising pneumonia $n=1$ ) and 15 healthy volunteers. None of the alveolar areas explored in the non-PAP ILD patients and healthy volunteers displayed the fluorescent globular material that was observed in our PAP patient. Therefore, these endomicroscopic characteristics appear strongly suggestive of the diagnosis of PAP during the bronchoscopy procedure. In a previous study [3], the authors were able to demonstrate that, in smokers, the strong fluorescence of the alveolar macrophages was related to the retention of tobacco tar in the alveolar fluid and macrophages. Tobacco tar is highly fluorescent at $488 \mathrm{~nm}$ excitation [10]. From this observation, it cannot be excluded that the visualisation of the globular fluorescent substance is related to the presence of the tobacco tar within the lipoproteinaceous PAP material. Therefore, the alveolar FCFM findings in nonsmoking PAP patients may differ from those described in our observation. However, because the majority of patients with primary PAP are active smokers [5, 11], this may represent a small limitation for the procedure, which should be further explored.

In conclusion, fluorescence bronchoalveoscopy is a minimally invasive and safe technique that may provide specific in vivo microimaging of PAP in real-time in smoking patients.

\author{
M. Salaün*,\#, F. Roussel ${ }^{\top}$, P-A. Hauss*, S. Lachkar* and \\ L. Thiberville*,\# \\ *Clinique Pneumologique, Rouen University Hospital, "Dept \\ of Pathology and Cytology, Rouen University Hospital, and \\ \#QuantIF Laboratory LITIS EA 4108, Faculty of Medicine and \\ Pharmacy, Rouen University, Rouen, France.
}

Correspondence: L. Thiberville, Clinique Pneumologique, Hôpital Charles Nicolle, CHU de Rouen, 1 rue de Germont, 76031 Rouen Cedex, France. E-mail: Luc.Thiberville@univ-rouen.fr

Statement of Interest: A statement of interest for L. Thiberville can be found at www.erj.ersjournals.com/misc/statements.dtl

\section{REFERENCES}

1 Thiberville L, Moreno-Swirc S, Vercauteren T, et al. In vivo imaging of the bronchial wall microstructure using fibered confocal fluorescence microscopy. Am J Respir Crit Care Med 2007; 175: 22-31.

2 Thiberville L, Salaun M, Lachkar S, et al. Confocal fluorescence endomicroscopy of the human airways. Proc Am Thorac Soc 2009; 6 : 444-449.

3 Thiberville L, Salaun M, Lachkar S, et al. Human in vivo fluorescence microimaging of the alveolar ducts and sacs during bronchoscopy. Eur Respir J 2009; 33: 974-985.

4 Maygarden SJ, Iacocca MV, Funkhouser WK, et al. Pulmonary alveolar proteinosis: a spectrum of cytologic, histochemical, and ultrastructural findings in bronchoalveolar lavage fluid. Diagn Cytopathol 2001; 24: 389-395.

5 Seymour JF, Presneill JJ. Pulmonary alveolar proteinosis: progress in the first 44 years. Am J Respir Crit Care Med 2002; 166: 215-235.

6 Trapnell BC, Whitsett JA, Nakata K. Pulmonary alveolar proteinosis. N Engl J Med 2003; 349: 2527-2539.

7 Holbert JM, Costello P, Li W, et al. CT features of pulmonary alveolar proteinosis. AJR Am J Roentgenol 2001; 176: 1287-1294.

8 Frazier AA, Franks TJ, Cooke EO, et al. From the archives of the AFIP: pulmonary alveolar proteinosis. Radiographics 2008; 28: 883-899.

9 Kitamura T, Uchida K, Tanaka N, et al. Serological diagnosis of idiopathic pulmonary alveolar proteinosis. Am J Respir Crit Care Med 2000; 162: 658-662.

10 Skold CM, Hed J, Eklund A. Smoking cessation rapidly reduces cell recovery in bronchoalveolar lavage fluid, while alveolar macrophage fluorescence remains high. Chest 1992; 101: 989-995.

11 Inoue $\mathrm{Y}$, Trapnell BC, Tazawa R, et al. Characteristics of a large cohort of patients with autoimmune pulmonary alveolar proteinosis in Japan. Am J Respir Crit Care Med 2008; 177: 752-762.

\section{Undiagnosed coeliac disease in patients with emphysema: a fortuitous association?}

\section{To the Editors:}

Chronic obstructive pulmonary disease (COPD) is characterised by progressive and poorly reversible airflow obstruction due to small airway disease and emphysema. Cigarette smoking is the major cause of COPD, but the disease also occurs in nonsmokers. In nonsmokers, environmental factors (e.g. second-hand smoking and inhalation of toxic gas), genetic factors (e.g. the rare $\alpha_{1}$-antitrypsin deficiency) and infectious factors (e.g. HIV infection) have been implicated in the pathogenesis of the disease. We report on two cases of
COPD with emphysema in nonsmokers with long-standing undiagnosed coeliac disease.

A 71-yr-old female was referred to our hospital (Hôpital Cochin, Assistance Publique-Hôpitaux de Paris, Paris, France) for dyspnoea and cough, which had evolved over 5 yrs. She had suffered from alternating diarrhoea and constipation for many years, ascribed to functional bowel disease. She had never been exposed to inhaled toxics and had never smoked. Examination revealed a body mass index (BMI) of $18 \mathrm{~kg} \cdot \mathrm{m}^{-2}$ and a chest computed tomography (CT) image revealed 